\title{
The power of model-to-crop translation illustrated by reducing seed loss from pod shatter in oilseed rape
}

\author{
Pauline Stephenson $^{1} \cdot$ Nicola Stacey $^{1} \cdot$ Marie Brüser $^{1,2} \cdot$ Nick Pullen $^{1} \cdot$ Muhammad llyas $^{1,3} \cdot$ Carmel O'Neill $^{1}$. \\ Rachel Wells ${ }^{1} \cdot$ Lars $_{\text {Østergaard }}{ }^{1}$ (1)
}

Received: 27 February 2019 / Accepted: 14 June 2019 / Published online: 20 June 2019

(c) The Author(s) 2019

Key message Elucidation of key regulators in Arabidopsis fruit patterning has facilitated knowledge-translation into crop species to address yield loss caused by premature seed dispersal (pod shatter).

Abstract In the 1980s, plant scientists descended on a small weed Arabidopsis thaliana (thale cress) and developed it into a powerful model system to study plant biology. The massive advances in genetics and genomics since then have allowed us to obtain incredibly detailed knowledge on specific biological processes of Arabidopsis growth and development, its genome sequence and the function of many of the individual genes. This wealth of information provides immense potential for translation into crops to improve their performance and address issues of global importance such as food security. Here, we describe how fundamental insight into the genetic mechanism by which seed dispersal occurs in members of the Brassicaceae family can be exploited to reduce seed loss in oilseed rape (Brassica napus). We demonstrate that by exploiting data on gene function in model species, it is possible to adjust the pod-opening process in oilseed rape, thereby significantly increasing yield. Specifically, we identified mutations in multiple paralogues of the INDEHISCENT and GA4 genes in $B$. napus and have overcome genetic redundancy by combining mutant alleles. Finally, we present novel software for the analysis of pod shatter data that is applicable to any crop for which seed dispersal is a serious problem. These findings highlight the tremendous potential of fundamental research in guiding strategies for crop improvement.

Keywords Arabidopsis thaliana $\cdot$ Brassica species $\cdot$ Model-to-crop translation $\cdot$ Fruit development $\cdot$ Pod shatter $\cdot$ Oilseed rape

Communicated by Venkatesan Sundaresan.

Pauline Stephenson, Nicola Stacey and Lars Østergaard have contributed equally to this work.

Electronic supplementary material The online version of this article (https://doi.org/10.1007/s00497-019-00374-9) contains supplementary material, which is available to authorized users.

Lars Østergaard

lars.ostergaard@jic.ac.uk

1 Department of Crop Genetics, John Innes Centre, Norwich Research Park, Norwich NR4 7UH, UK

2 Present Address: London, UK

3 Present Address: Department of Biological Sciences, Faculty of Basic and Applied Science, International Islamic University, Islamabad, Pakistan

\section{Introduction}

Food security is being challenged worldwide due to population increase and climate change (Tilman et al. 2011; Fisher et al. 2014; Hunter et al. 2017). It is therefore crucial that plant scientists explore every opportunity for enhancing the efficiency with which crops are grown. Advances in plant molecular biology, genetics and genomics have over the last 3 decades led to an explosion in our understanding of specific biological processes, the function of individual genes and genome dynamics during plant development. This wealth of information holds an incredible potential to be exploited for translation into improved and sustainable crop production (Boden and Østergaard 2019).

More research funds have been invested into understanding the biology of Arabidopsis than to any other plant species in the belief that many processes controlling all aspects of plant development are conserved across the plant kingdom. Attempts to transfer knowledge from Arabidopsis to 
crops seem a valid test of this assumption. Since members of the Brassica genus are the closest crop relatives to Arabidopsis, model-to-crop translation between the Arabidopsis and Brassica crops provides potentially huge opportunities. The aim of this paper is to provide an illustrative example of how understanding the molecular details of a specific process during plant development can be used to address a serious barrier to more efficient crop production.

\section{Morphological conservation of fruit structures in Arabidopsis and Brassica species}

B. napus along with other important Brassica crops is a member of the Brassicaceae family, which also contains Arabidopsis. The close evolutionary relationship between them is reflected in the conservation of the genome structure with segments of genetically linked loci of the B. napus genome corresponding to regions of the Arabidopsis genome exhibiting a high level of synteny (Parkin et al. 2005).
Moreover, B. napus and other Brassica species have very similar plant architecture and organ morphology to Arabidopsis (Fig. 1), and it is therefore plausible that knowledge of significant biological processes obtained in Arabidopsis could be used to improve the performance of oilseed rape.

Pod shatter is a term used for the preharvest fruit opening and seed dispersal of oilseed rape (B. napus) leading to yield loss. The similarities in fruit morphology between Arabidopsis and Brassica species (Fig. 1) suggest that the underlying patterning mechanism is conserved. It is therefore possible that knowledge on fruit opening in Arabidopsis can be exploited to address the problem of pod shatter in oilseed rape. After fertilisation at developmental stage 13 [stages defined for Arabidopsis flower development in Smyth et al. (1990) and conserved in Brassicas as described in Girin et al. (2010)], Brassicaceae fruits elongate and form a number of specialised tissues including valves (also known as pod walls), a central replum and valve margins that form at the valve/replum borders where fruit opening will take place (Fig. 1c, g). This process is the result of precise tissue- and cell-type specification allowing the separation of
Fig. 1 Conserved plant architecture and pod morphology between Brassica $(\mathbf{a}-\mathbf{d})$ and Arabidopsis (e-h). a 8-weekold Brassica rapa plant. b mature pod (stage 17) from B. rapa. c SEM of stage 15 B. rapa pod. d wholemount image of dehiscing $B$. napus pod (stage 19). e 5-week-old Arabidopsis plant. f mature pod (stage 17) from Arabidopsis. g SEM of stage 17 Arabidopsis pod. h SEM of dehiscing Arabidopsis fruit (stage 19). i Schematic cross section of pod from Arabidopsis/Brassica with valves in green, replum in brown, lignified layer in red and separation layer in light blue. A simplified regulatory network is indicated above the close-up on valve margin region. Blue arrowheads in $\mathbf{c}$ and $\mathbf{g}$ indicate position of valve margins. $\mathrm{V}$ valve, $\mathrm{R}$ replum, $\mathrm{L}$ lignified layer, S separation layer. Scale bars correspond to $100 \mu \mathrm{m}$ in $\mathbf{g}$, $500 \mu \mathrm{m}$ in $\mathbf{c}$ and $\mathbf{h}, 1 \mathrm{~mm}$ in $\mathbf{f}$, $5 \mathrm{~mm}$ in $\mathbf{d}, 1 \mathrm{~cm}$ in $\mathbf{b}, 2 \mathrm{~cm}$ in $\mathbf{e}$ and $10 \mathrm{~cm}$ in $\mathbf{a}$
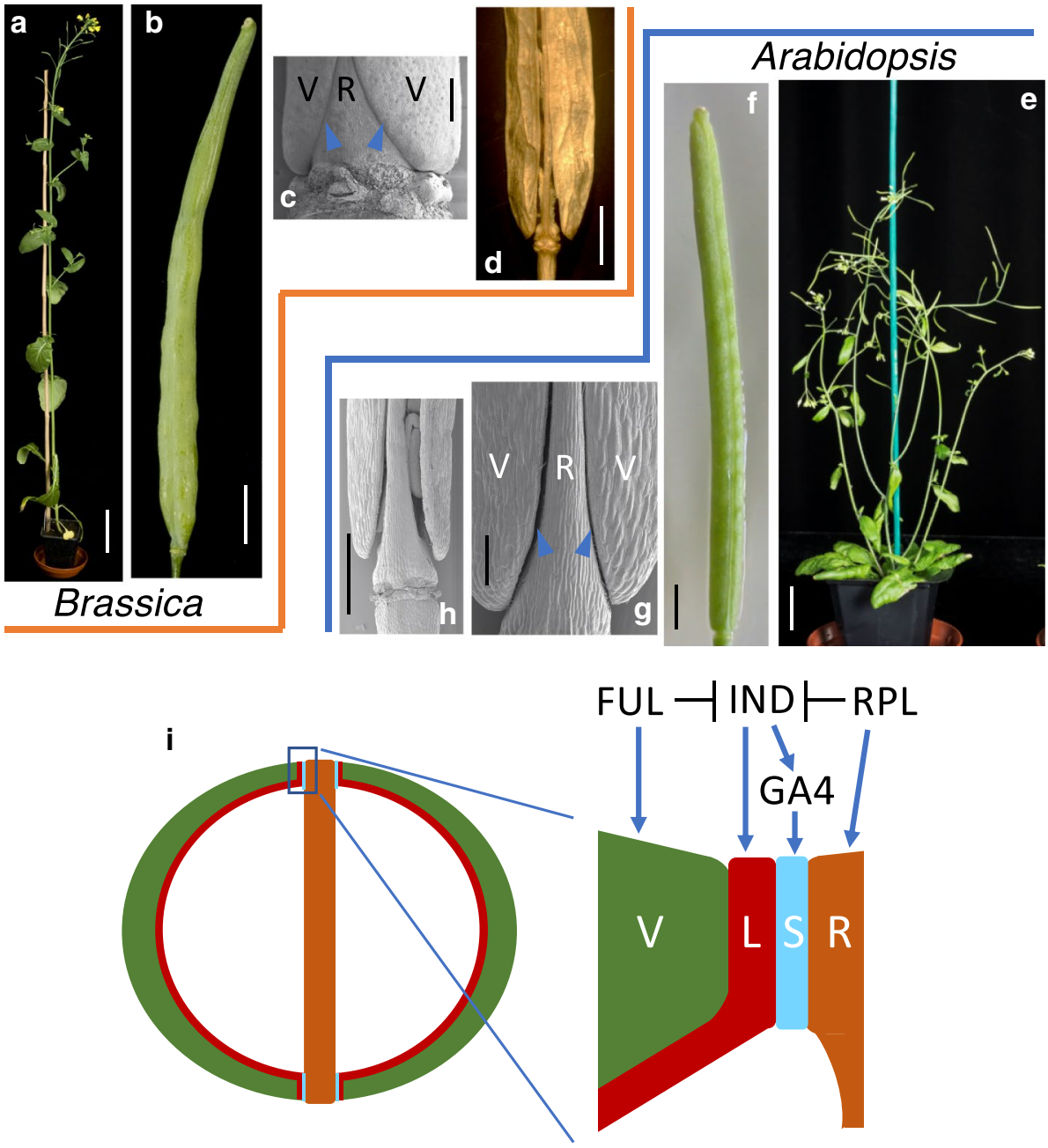
the valves from the replum and leading to the timely dispersal of mature seeds (Fig. 1d, h; Roberts et al. 2002). The valve margins are composed of two distinct cell types, a lignified layer and a separation layer (Fig. 1i). Upon fruit maturation, cells in the valve margins mediate fruit opening through degradation of the pectin-rich separation layer by secreting polygalacturonase enzymes (Ogawa et al. 2009; Petersen et al. 1996; Degan et al. 2001; Spence et al. 1996).

Although efficient seed dispersal is an advantage for plants growing in the wild, unsynchronised pod shatter of oilseed rape causes average annual losses above $10 \%$ of harvest (Price et al. 1996) which can exceed 70\% under adverse weather conditions (de la Pasture 2018). Controlling pod shatter is in fact such an important trait for oilseed rape yield increase, and many other traits focused around plant architecture are aimed at reducing pod shatter-mediated seed loss in the field (Morgan et al. 2000).

\section{Conservation of genetic and hormonal activities in Brassicaceae fruit development}

Setting up the overall patterning process of the Brassicaceae fruit is a prerequisite for proper development. Several of the key genetic factors from Arabidopsis have been identified and the interactions between them established (Fig. 1i). For a comprehensive description of all known components, see Dinneny et al. (2005). Here, we focus on the INDEHISCENT (IND) gene, which encodes a basic helix-loop-helix (bHLH) transcription factor that is essential for valve margin formation (Liljegren et al. 2004). The highly specific expression of the IND gene in valve margin tissue is ensured by the repressing activities of the FRUITFULL (FUL) gene in the valves and the REPLUMLESS (RPL) gene in the replum (Ferrándiz et al. 2000; Roeder et al. 2003; Dinneny et al. 2005).

We previously found that this genetic network interacts with activities of the phytohormones auxin and gibberellin (GA) to ensure proper fruit patterning in Arabidopsis (Sorefan et al. 2009; Arnaud et al. 2010; Girin et al. 2011). In wild-type fruits, both local depletion of auxin and biosynthesis of GA are required at the valve margin for specification of the separation layer where fruit opening takes place. IND mediates these events by directly regulating genes involved in both processes. The GA4 gene in Arabidopsis encodes an enzyme (GA3OX1) that mediates the final step in the biosynthesis of active gibberellins $\left(\mathrm{GA}_{1}\right.$ and $\left.\mathrm{GA}_{4}\right)$ (Talon et al. 1990), and our previous work revealed that IND binds to and directly induces expression of the GA4 gene (Arnaud et al. 2010). The production of gibberellin leads to derepression of another key regulator of valve margin formation, ALCATRAZ (Rajani and Sundaresan 2001) by promoting degradation of DELLA growth repressors (Arnaud et al. 2010).
Yield of oilseed rape could be significantly improved by controlling pod shatter. However, very little genetic diversity exists within $B$. napus germplasm presumably because allelic variation in genes controlling pod shatter was limited when B. napus emerged (Raman et al. 2014). To overcome pod shatter in oilseed rape, it therefore seems a more promising approach to directly target the genes known to be involved. Indeed, studies of fruit development in Arabidopsis and diploid Brassica species have provided directions for achieving pod shatter resistance by translation of knowledge on gene function. For example, we have shown that ectopic expression of the Arabidopsis FUL gene under the CaMV $35 \mathrm{~S}$ promoter leads to a complete loss of shattering in $B$. juncea as previously observed in Arabidopsis (Fig. 2; Østergaard et al. 2006; Ferrándiz et al. 2000). Although this result demonstrates that it is possible to transfer knowledge from the model system to Brassica crops, total encapsulation of the seeds in a fruit with no remaining ability to dehisce is undesirable for oilseed rape production, as significant losses would be incurred when attempting to retrieve the seeds. Subsequently, we used $B$. rapa (a progenitor of $B$. napus) as a system to directly target an IND homologue (BraA.IND.a), which exists as a single-copy gene in the $B$. rapa genome. Using a $B$. rapa TILLING population (Stephenson et al. 2010), we demonstrated that strong braA.ind mutant alleles are indehiscent (Fig. 2b) and that it was possible to obtain weaker alleles in which valve margin tissue was only partially lost (Girin et al. 2010). This intriguing result showed that it is feasible to fine-tune the level of shatter resistance in Brassica fruits by adjusting the activity of IND homologues. We also identified a functional IND homologue, BolC.IND, in the other B. napus progenitor, B. oleracea (Girin et al. 2010). Moreover, in agreement with $G A 4$ being positively regulated by $I N D$ as described above, we also demonstrated that a gene-edited line in which two BolC.GA4 paralogues were knocked out resulted in indehiscent (i.e. pod shatter resistant) pods (Fig. 2b; Lawrenson et al. 2015).

In this study, we obtain and analyse mutant combinations of IND and GA4 paralogous genes of the allotetraploid $B$. napus (oilseed rape). Our results provide the final stage of a model-to-crop translation pipeline from fundamental discoveries in a model system via proof-of-concept in diploid Brassica species to validation in oilseed rape, thereby revealing the power of such strategies for crop improvement.

\section{Materials and methods}

\section{Plant material and plant growth}

B. napus var. Cabriolet wild type and mutants were sown in F9 pots containing F1 compost and then grown at long day $(16 \mathrm{~h} \mathrm{light} / 8 \mathrm{~h}$ dark $)$ at $18{ }^{\circ} \mathrm{C}$ day and $12{ }^{\circ} \mathrm{C}$ night 
a A. thaliana

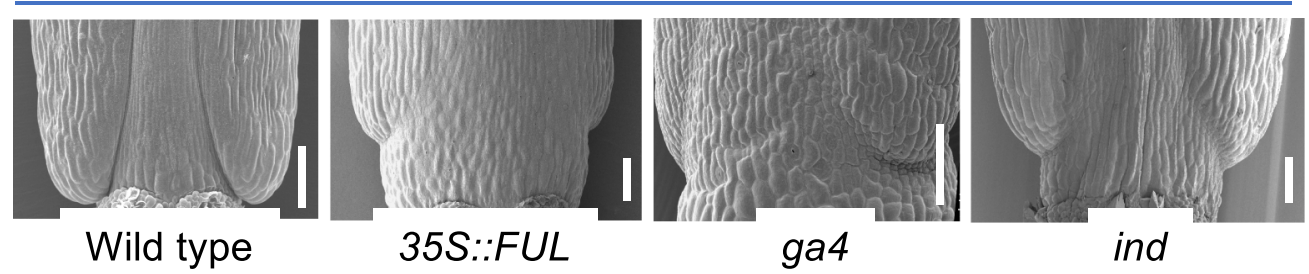

b

B. juncea

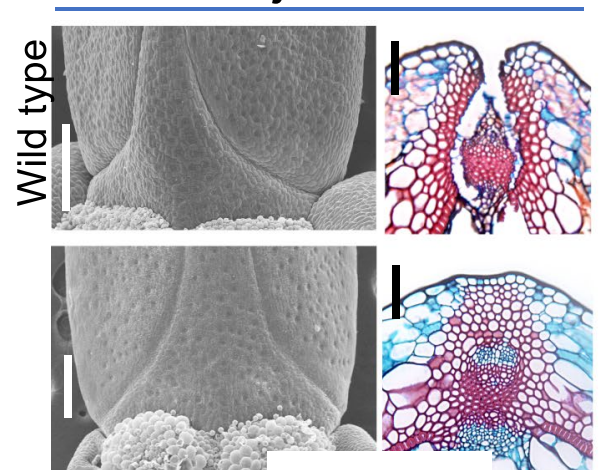

35S::FUL
B. rapa

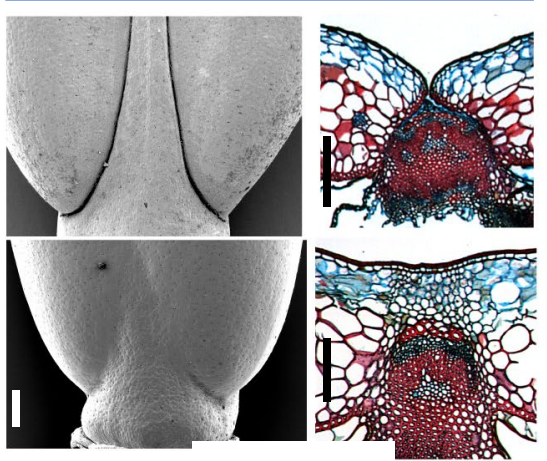

braA.ind
B. oleracea
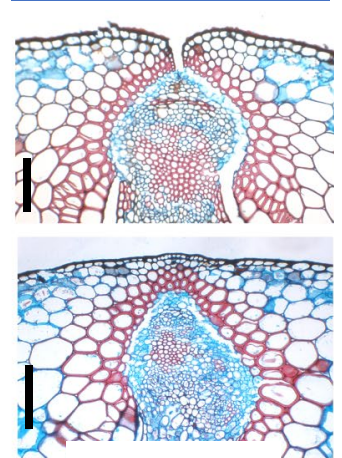

bolC.ga4
Fig. 2 Effect of gain- and loss-of function of genes involved in valve margin formation. a SEM images of the bases of fruits at stage 17 from Arabidopsis wild type, $35 S:: F U L$, ga4 and ind mutants. b SEM images and staining of cross sections are shown for the indicated Brassica species. Sections are from the middle of the fruits where the

temperature. After 6-week vernalisation at $5{ }^{\circ} \mathrm{C}$, plants were potted on into 1-L pots containing John Innes Number 2 compost. They were then grown in a glasshouse with longday conditions at $18{ }^{\circ} \mathrm{C}$ day and $12{ }^{\circ} \mathrm{C}$ night temperature.

\section{Phylogenetic analyses}

Brassica IND and GA4 sequences were identified from EnsemblPlants by using BLAST with the Arabidopsis IND and GA4 protein sequences, respectively. Alignments were produced using the Clustal Omega software from EMBLEBI, and phylogenies were produced using the phylogeny.fr software package (Dereeper et al. 2008).

\section{Random impact test (RIT) assay}

Pod shatter resistance was quantified by the RIT assay first described in Morgan et al. (1998). Fruits were harvested at stage 19 (stages defined in Smyth et al. 1990; Girin et al. 2010), when they were fully dry, but unopened and placed in portions of 20 into small perforated bags, which allow moisture exchange. Bags of fruits were placed in a dehumidification chamber and equilibrated to $50 \%$ humidity for $>48 \mathrm{~h}$. Twenty undamaged fruits were used in each separate assay, and assays replum is narrower. Upper row displays images from wild-type fruits at stage 17, whereas stage 17 fruits from the indicated mutant genotypes are shown in the lower row. Scale bars correspond to $100 \mu \mathrm{m}$ for $A$. thaliana SEMs and B. juncea and B. oleracea sections, $200 \mu \mathrm{m}$ for $B$. rapa sections and $250 \mu \mathrm{m}$ for $B$. juncea and B. rapa SEMs

were performed in triplicates (i.e. 60 fruits required per data point). Fruits were placed together with six $8-\mathrm{mm}$ steel balls in a $20-\mathrm{cm}$-diameter cylindrical container. The container was shaken in the Random Impact Test machine (produced at the John Innes Centre) at a frequency of $4.98 \mathrm{~Hz}$ and a stroke length of $51 \mathrm{~mm}$ for 8 -s intervals until all the fruits were dehisced. After each interval, fruits were examined and the number of intact fruits remaining was counted. Fruits that had released any of their seeds were considered broken.

\section{podshatteR software}

Calculation of $\mathrm{RIT}_{50}$ values was performed using the podshatteR software developed in this study. A description and step-by-step guide are provided in the Online Resource material. Briefly, this software provides an upgrade in curve fitting compared to previous reports (Morgan et al. 1998). Following the initial plotting of data by podshatteR, data were visually inspected to identify any errors or ambiguities. Curve fitting was then performed using an exponential decay function to keep the number of free parameters minimal, i.e.

Value $=A \exp (-b \times$ Time $)$ 
where $A$ is the intercept (which should be similar to the starting number of pods) and $b$ is the decay rate. Through propagation of errors, confidence intervals were computed for each sample. The half-life was then calculated for each sample as the value at which half the initial number of pods has shattered, following the model fit.

\section{Tissue staining}

Tissues were fixed for $6 \mathrm{~h}$ in an FAA solution $(3.7 \%$ formaldehyde, $5 \%$ acetic acid, 50\% ethanol) and subsequently dehydrated through an ethanol series. The tissues were cleared with Histoclear (National Diagnostics) and embedded in paraffin. An RM2255 rotary microtome (Leica) was used to make $8 \mu \mathrm{m}$ transversal stem sections.

After deparaffinisation, sections were stained with an Alcian Blue $8 \mathrm{Gx} /$ Safranin-O solution $(0.05 \%$ Alcian Blue $8 \mathrm{Gx}$ and $0.01 \%$ Safranin-O in $0.1 \mathrm{M}$ acetate buffer [pH 5.0]) as described in Østergaard et al. (2006). Sections were examined under light microscopy.

\section{Results and discussion}

\section{Obtaining and characterising B. napus IND mutants}

We identified two sequences from B. napus with $>90 \%$ identity to the diploid Brassica IND genes (alignment in Online Resources 1). Based on the genome sequence of the B. napus variety Darmor- $b z h$ (Chalhoub et al. 2014), the accession numbers are BnaC03g32180D and BnaA03g27180D, which places them on chromosomes $\mathrm{C} 3$ and $\mathrm{A} 3$, respectively. These genes are identical to the $B$. napus orthologues identified by Braatz et al. (2018), and their annotated chromosomal locations are in agreement with our previous mapping of two IND genes in the Tapidor/Ningyou7 doubled haploid mapping population (Qiu et al. 2006). For simplicity, we will henceforth use the names previously assigned to these genes, BnaC.IND.a and BnaA.IND.a (Braatz et al. 2018). A phylogenetic analysis revealed that instead of being most closely related to each other, the two B. napus IND-like sequences cluster separately with BraA.IND.a and BolC. IND. $a$ from the diploid progenitors (Fig. 3a). Together, these results demonstrate that the two IND sequences identified in $B$. napus originate from each of the two diploid progenitors (B. rapa and B. oleracea) and are not caused by duplication after the hybridisation event that formed B. napus.

In order to obtain loss-of-function bnaA.ind. $a$ and bnaC. ind. $a$ mutants, we first designed paralogue-specific amplicons for the two B. napus IND genes (Online Resource 2a, b) and subsequently screened the $B$. napus TILLING resource available in the variety Cabriolet from the RevGenUK platform at the John Innes Centre (www.revgenuk.ac.uk). We did not identify any mutations that would generate premature stop codons; however, out of the allelic series that were obtained, we chose four mutant alleles for each paralogue for further studies (Fig. 3b). These alleles were chosen based on the nature of the amino acid change (e.g. charge, hydrophobicity, size) and conservation among IND from Arabidopsis (see alignment in Online Resource 1). For example, charge changes include the D16N and G53R mutations, the T146I mutation changes hydrophobicity and both G53R and R95H mutations involve changes in amino acid size.

In agreement with functional redundancy of the two $B$. napus IND genes, we did not observe any phenotypic defects in single mutants (Online Resource 2c, d). Crosses were therefore performed in order to obtain double mutants. We performed all combinations of crosses resulting in 16 different double-mutant lines (Online Resource 3a).

The mutant lines did not display the same dramatic effects on valve margin formation as the knock-out and overexpression lines shown in Fig. 2. However, given that complete indehiscence will not be desirable for oilseed rape farmers in terms of recovering seeds, fine-tuning IND and GA4 activities through pairing of mutant alleles may provide levels of dehiscence better suited for yield increase. To quantify pod shatter resistance of fruits from the B. napus mutant lines generated here, we used the Random Impact Test (RIT) assay first developed by Morgan et al. (1998). The RIT applies mechanical force to mature and dry Brassica fruits by shaking them in a container in the presence of metal ball bearings for a certain amount of time (Online Resource $4 a-c)$. The time it takes for half of the fruits in the container to break open is taken as a measure of their level of pod shatter resistance (Morgan et al. 1998) and referred to here as the $\mathrm{RIT}_{50}$ value (Online Resource 4d). To better analyse these data, we developed the software package, podshatteR. Based within the free R software environment, it provides a simple GUI with a step-by-step process for improved data quality analysis, curve fitting and therefore a more precise quantification of the RIT $_{50}$ value (Online Resource file).

For the bnaA.ind.a bnaC.ind.a double mutants, we obtained fruits suitable for the RIT assay for 13 out of the 16 combinations and tested them in comparison with the wild-type Cabriolet variety, which is the background for the TILLING population. For simplicity, we will refer to these double mutants by their amino acid substitution as in Fig. 3c where the first annotation refers to the substitution on the A genome and the second on the $\mathrm{C}$ genome. The graph in Fig. $3 \mathrm{c}$ shows a wide range of shatter resistance from lines such as $G 53 R / T 146 I$ that exhibit less resistance than wild type to $M 70 I / T 146 I$ and $D 16 N / R 95 H$ that are significantly more resistant than wild type. Three combinations produced fruits that were largely infertile and therefore not included in the analysis (Online Resource 3a). This may be due to background mutations caused by EMS at other loci that affected 
Fig. 3 Identifying and characterising bna.ind mutants. a Phylogenetic analysis of Arabidopsis and Brassica IND protein sequences. HEC3 is HECATE3, which is the closest homologue of IND in Arabidopsis and used as an outgroup in this analysis. Scale bar indicates substitutions per site. b Schematic of IND protein divided into its three domains: the INDspecific (IS), HECATE (HEC) and basic helix-loop-helix (bHLH) domains. Position and nature of missense mutations are shown with four from the A genome (top) and four from the $\mathrm{C}$ genome (bottom). $\mathbf{c}$ RIT half-lives $\left(\mathrm{RIT}_{50}\right)$ plotted for the wild-type variety 'Cabriolet' and the combinations of bna. ind double mutants indicated in the graph with substitutions on the A genome in front of substitutions on the $\mathrm{C}$ genome. Error bars indicate standard error. $* p<0.05 ; * * p<0.01$; $* * * p<0.001$

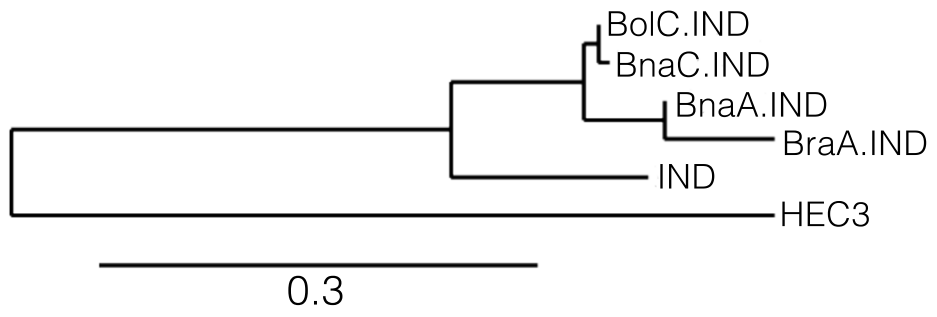

b

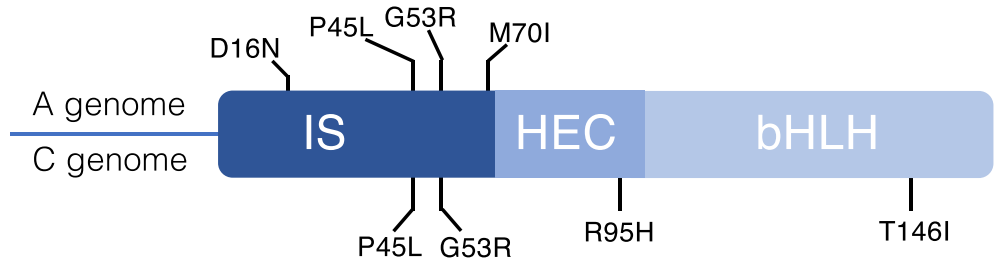

C 2.5

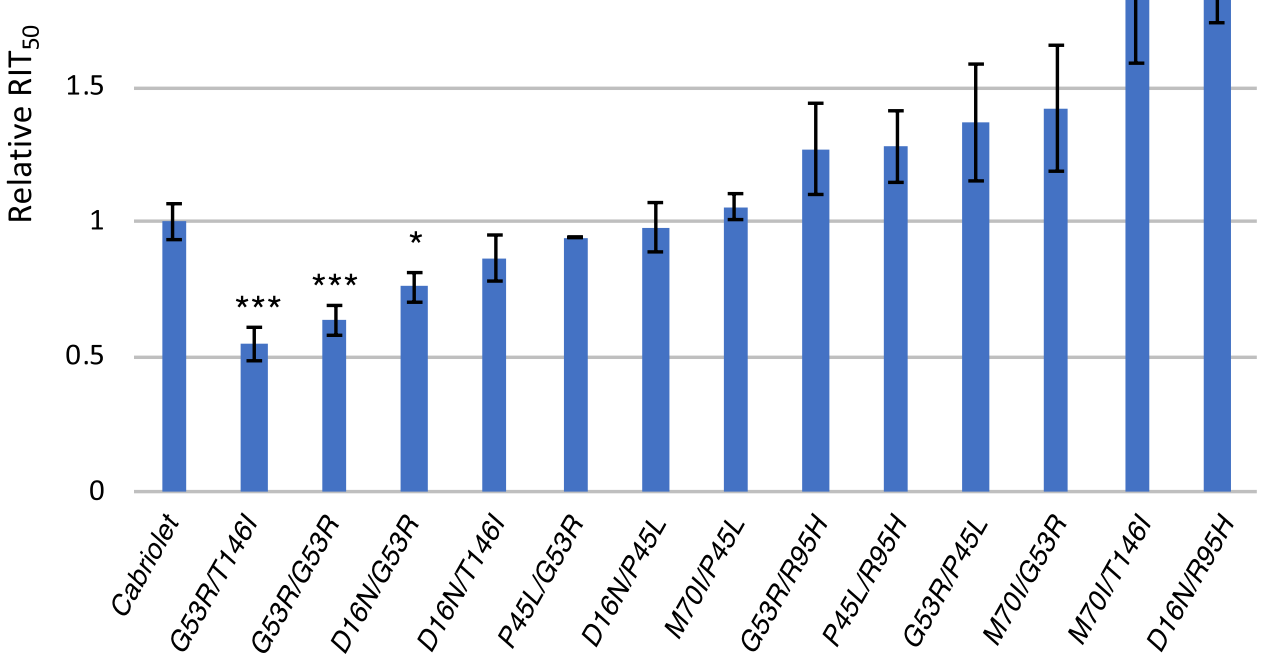

the development of, for example, pollen or stigma or other tissues important for efficient fertilisation.

It is not clear which residue mutations provide the effect as combinations with each of the individual mutations in the resistant lines also found in combinations with less resistance (Fig. 3c). The significant increased shattering of $G 53 R /$ $T 146 I$ and $G 53 R / G 53 R$ is intriguing, and it is possible that also this phenotype is due to complications caused by background mutations. On the other hand, Bra.IND proteins are members of the bHLH family of transcription factors who are known to function as dimers (Amoutzias et al. 2008). It is therefore possible that certain combinations lead to increased compatibility in heterodimer formation that may enhance the activity of the complex. To prove this will require further analysis.
Here, we focused on the lines with increased shatter resistance and analysed sections of the $D 16 \mathrm{~N} / \mathrm{R} 95 \mathrm{H}$ and $M 70 I / G 53 R$ at different developmental stages late in fruit development (Fig. 4; Online Resource 3b). Although the differences are subtle compared to the effects seen in full ind knockouts (Fig. 2), there are features, which may explain the increased shatter resistance in the mutant lines. At stage 16, valve margin cells are becoming apparent in wild-type fruits as small light blue cells. The definition of these cells is less pronounced in the two best performing mutant combinations (Fig. 4, left; Online Resource 3b). At stage 17B, when lignification is visible, the light blue separation layer appears wider in the wild type (Fig. 4, middle). Moreover, at full maturity (stage 17c), valves detach completely in the wild-type fruits, but remain 
Fig. 4 Tissue sections of fruits from Cabriolet (wild type) and the bnaA.ind.a-D16N bnaC. ind. $a-R 95 H$ double mutant $(D 16 N / R 95 H)$ at the indicated developmental stages. Red arrowheads point to separation layer primordium cells in Cabriolet. Black arrowheads indicate lack of complete separation in the $D 16 N / R 53 H$ double mutant. Scale bars correspond to $100 \mu \mathrm{m}$

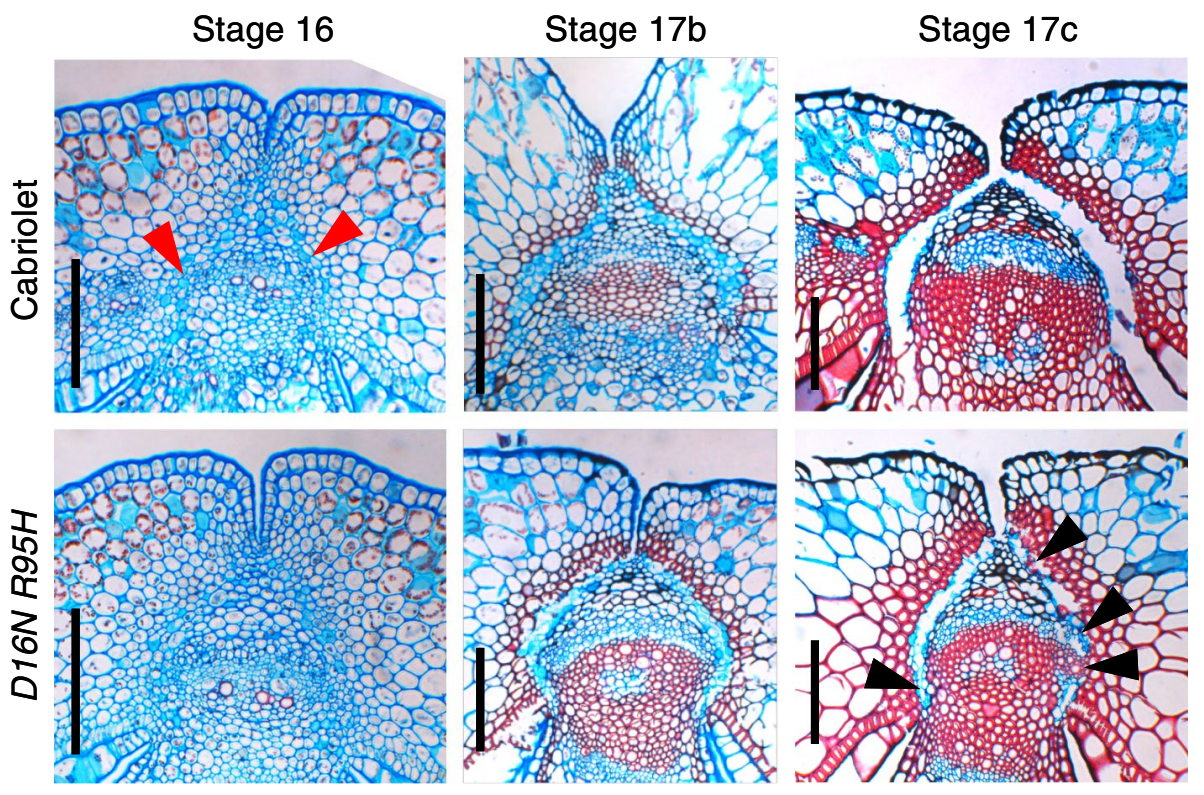

partially connected in the mutants (Fig. 4, right; Online Resources 3b).

Taken together, these data show that by obtaining an allelic series of mutations in the Bna.IND genes, it is possible to make combinations in order to fine-tune the level of pod shattering.

\section{Obtaining B. napus GA4 mutants}

We have previously described how knocking out two GA4 paralogues in B. oleracea by CRISPR/Cas9 led to the production of pod-shatter-resistant siliques (Lawrenson et al. 2015). Moreover, the plants displayed a dwarf architecture in agreement with the phenotype of ga4 mutants in Arabidopsis (Chiang et al. 1995).

In parallel with the isolation of $B$. napus ind mutants, we attempted to obtain mutants in the B. napus $G A 4$ orthologues. The $B$. napus genome contains four $G A 4$ paralogues (BnaA06g10250D, BnaA09g57140D, BnaC05g11920D and BnaC08g38810D), which we rename for simplicity as BnaA6.GA4, BnaA9.GA4, BnaC5.GA4 and BnaC8.GA4, respectively. The encoded proteins show a very high level of identity to each other and to GA4 from Arabidopsis of 85-90\% (see alignment in Online Resource 5). Nevertheless, a phylogenetic analysis demonstrated that each of these is most closely related to the paralogues on the corresponding chromosomes in the $B$. rapa and $B$. olerace $a$ diploids (Fig. 5a). This analysis demonstrates that B. napus GA4 paralogues are conserved from the diploid progenitors and have not undergone any additional duplication or gene loss since the hybridisation of the diploids which formed the allotetraploid B. napus.
We designed specific amplicons for each of the four paralogues as described for the B. napus IND alleles above. By screening the $B$. napus Cabriolet TILLING population, we obtained allelic series of all. For three of these $G A 4$ genes, at least one allele gave rise to a premature stop codon in the 5 ' half of the coding region (Fig. 5b, Online Resource 5) and we decided to focus on these for the subsequent analyses. As expected, due to redundancy among the paralogues, none of the single mutants led to any phenotypic defects (Online Resource 6). Hence, all three combinations of double mutants were produced by crossing and homozygous lines obtained.

The effect on valve margin development in ga4 mutants in Arabidopsis is specific to the separation layer, and the level of indehiscence of ga4 fruits is therefore not as pronounced as for fruits from the ind mutant that have lost both separation layer and lignified layer (Arnaud et al. 2010). Rather than developing a large allelic series using combinations of missense mutations, we focussed on the nonsense mutations, which gave rise to premature stop codons in three of the $B$. napus GA4 paralogues.

In a RIT analysis, we found that the combination of the bnaA9.ga4 bnaC5.ga4 double exhibited the largest effect with shatter resistance $\sim 2.5$-fold above wild type with significant increase in shatter resistance observed for both directions of crossing, i.e. when pollen from the $C 5$ allele was applied to $A 9$ stigma and vice versa (Fig. 5c). In agreement with the increased resistance, cross sections of stage-17C fruits from the $C 5 x A 9$ double mutant exhibited reduced separation at the valve margin compared to wild type (Fig. 5d).

In both examples shown here, 2-3-fold higher shatter resistance was obtained. Interestingly, this is in the same range as the best performing bna.ind double mutant line 
Fig. 5 Identifying and characterising B. napus ga4 mutants. a Phylogenetic analysis of Arabidopsis and Brassica IND protein sequences. AtGA3OX2 was used as an outgroup in this analysis. Scale bar indicates substitutions per site. b Schematic of the four GA4 proteins indicating position of nonsense mutations in the paralogues on chromosomes A6, A9 and C5. c RIT ${ }_{50}$ values plotted for 'Cabriolet' ( $\mathrm{Cab}$ ) and reciprocal crosses of the double mutant of paralogues on chromosomes A9 and C5. Error bars indicate standard error. $* * p<0.01$. d Tissue sections of fruits from Cabriolet (wild type) and the bnaA09.ga4 bnaC05. ga4 (C5×A9) double mutant. Black arrowheads indicate lack of complete separation in the $C 5 \times A 9$ double mutant. Scale bars correspond to $100 \mu \mathrm{m}$ a

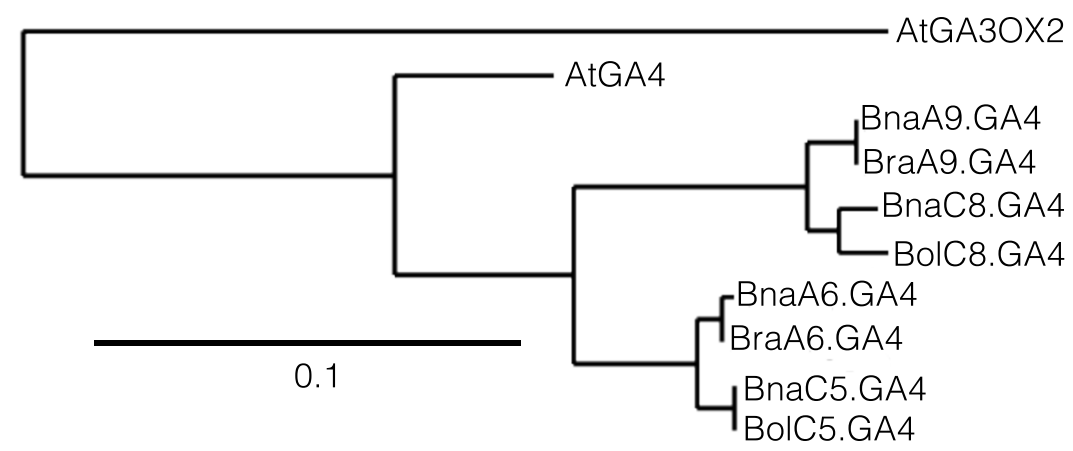

b

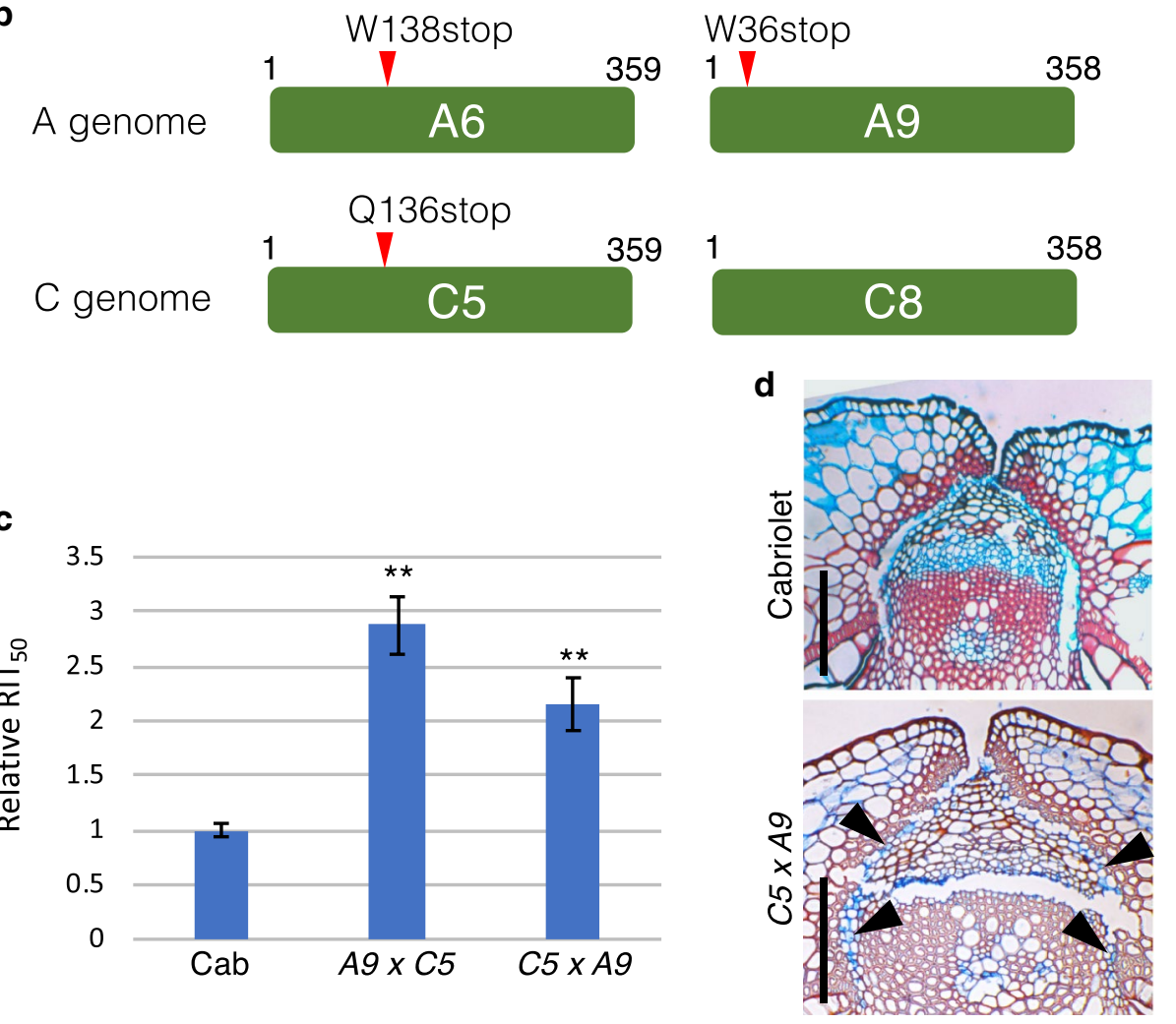

obtained in the 'Express' variety ( 3-fold increase compared to wild type) (Braatz et al. 2018). Also, a 2.5-fold improvement was obtained in the InVigor hybrid line developed by Bayer in which the two bna.ind mutants are heterozygous knockouts (European Patent Specification 2008).

In conclusion, the RIT $_{50}$ data for certain of the mutant combinations obtained here revealed a significant increase in resistance compared to wild type. In particular, the bna. ind D16N/R95H and bnaA9.ga4 bnaC5.ga4 mutants led to resistance that is comparable to previous reports (Braatz et al. 2018; European Patent Specification 2008) and therefore hold promising potential for inclusion in future breeding programmes. We have recently demonstrated that higher temperature accelerates pod shatter in fruits from a range of Brassicaceae species including Arabidopsis and B. napus. Our experiments revealed this effect to be mediated at least partially by upregulation of the IND gene (Li et al. 2018). It is therefore plausible that the lines generated here will maintain their high shatter resistance independent of temperature increase, potentially leading to an even higher relative improvement compared to the Cabriolet wild type.

\section{Perspectives}

In the last 3 decades, investment in research to understand the growth and development of Arabidopsis thaliana has been second to none in the plant kingdom. The amount of knowledge on, for example, gene regulation, hormone dynamics, metabolism and disease resistance, emerging from this investment has been impressive. Nevertheless, politicians, funders and taxpayers have a right to question whether this enormous attention to a small weed is justified. 
Scientifically, discoveries in Arabidopsis have no doubt led to a significantly increased basic understanding of plant biology and more widely biology in general. Whilst this may not have immediate societal impact, it contributes to a fundamental part of being human-i.e. a continued strive towards understanding the world we live in.

The model-to-crop project described here provides a reassuring example that, in terms of crop improvement, the focus on Arabidopsis has been a valuable investment. The results in the $B$. napus crop are based purely on a pipeline originating from fundamental discoveries in Arabidopsis going back $>20$ years since the first gene was discovered (FUL; Gu et al. 1998). In fact, identification of the key regulators would almost certainly not have been achievable directly in a polyploid crop, such as B. napus. The forward screens involved and requirements to overcome redundancy of paralogues would simply make it unfeasible to identify the key regulators and elucidate the genetic and hormonal networks in B. napus, even with the advanced gene-editing and genomics technologies available today.

The strategy for addressing pod shatter in oilseed rape by the approach described here has depended entirely on a translational approach. This work therefore demonstrates how knowledge obtained in a model system can be successfully transferred for use in crop improvement programmes. It should therefore serve as an encouraging example for scientists, the industry and funding agencies to get involved and support such undertakings.

Vast amounts of genetics and genomics resources are becoming available directly in crop species such as wheat (Uauy 2017), rice (Wing et al. 2018), maize (Nannas and Dawe 2015) and also in Brassica species (Lawrenson et al. 2015; Chalhoub et al. 2014). Therefore, addressing fundamental questions is no longer restricted to Arabidopsis or other relatively simple genetic systems. Scientists can now test hypotheses directly in crops to an extent that has not previously been possible, which will lead to an acceleration in crop improvement. Nevertheless, the small size, simple genetics and short generation time will ensure that Arabidopsis thaliana and other model plants remain attractive and important model systems for future discoveries with the potential to save billions from starvation and this no doubt will expand our fundamental understanding of biology on the planet.

Author contribution statement PS, NS and LØ designed the research. NP developed podshatteR software. PS, NS, MB, MI, CO and RW performed experiments. L.Ø. wrote the manuscript, and all authors commented on it.

Acknowledgements We thank the following science support services at the John Innes Centre: RevGenUK TILLING platform, Bio-imaging,
Photography and Horticulture for skilful assistance. This work was supported by Grants BB/J533055/1 (Follow-on Fund), BB/I017232/1 (Crop Improvement Research Club) and BB/P003095/1 (Strategic LoLa) to L.Ø. from the Biotechnological and Biological Sciences Research Council and by the Institute Strategic Programme Grants BB/J004553/1 and BB/P013511/1 to the John Innes Centre.

Open Access This article is distributed under the terms of the Creative Commons Attribution 4.0 International License (http://creativeco mmons.org/licenses/by/4.0/), which permits unrestricted use, distribution, and reproduction in any medium, provided you give appropriate credit to the original author(s) and the source, provide a link to the Creative Commons license, and indicate if changes were made.

\section{References}

Amoutzias GD, Robertson DL, van de Peer Y, Oliver SG (2008) Choose your partners: dimerization in eukaryotic transcription factors. Trends Biochem Sci 33:220-229

Arnaud N, Girin T, Sorefan K, Fuentes S, Lawrenson T, Wood T, Sablowski R, Østergaard L (2010) Gibberellins control fruit development in Arabidopsis thaliana. Genes Dev 24:2127-2132

Boden SA, Østergaard L (2019) How can developmental biology help feed a growing planet? Development. https://doi.org/10.1242/ dev. 172965

Braatz J, Harloff HJ, Emrani N, Elisha C, Gorb SN, Jung C (2018) The effect of INDEHISCENT point mutations on silique shatter resistance in oilseed rape (Brassica napus). Theor Appl Genet 131:959-971

Chalhoub B, Denoeud F, Liu S, Parkin IAP, Tang H, Wang X, Chiquet $\mathrm{J}$ et al (2014) Early allopolyploid evolution in the post-neolithic Brassica napus oilseed genome. Science 345:950-953

Chiang HH, Hwang I, Goodman HM (1995) Isolation of the Arabidopsis GA4 locus. Plant Cell 7:195-201

de la Pasture L (2018) Pod shatter-shattering implications for pod pop. Crop Production Magazine. http://www.cpm-magazine. co.uk/2018/05/02/pod-shatter-shattering-implications-pod-pop/. Accessed 19 June 2019

Degan FD, Child R, Svendsen I, Ulvskov P (2001) The cleavable $\mathrm{N}$-terminal domain of plant endopolygalacturonases from clade $\mathrm{B}$ may be involved in a regulated secretion mechanism. J Biol Chem 276:35297-35304

Dereeper A, Guignon V, Blanc G, Audic S, Buffet S, Chevenet F, Dufayard JF, Guindon S, Lefort V, Lescot M, Claverie JM, Gascuel O (2008) Phylogeny.fr: robust phylogenetic analysis for the non-specialist. Nucl Acids Res 36(Web Server issue):W465-W469

Dinneny JR, Weigel D, Yanofsky MF (2005) A genetic framework for fruit patterning in Arabidopsis thaliana. Development 132:4687-4796

European Patent Specification (2008) Brassica plant comprising a mutant indehiscent allele. EP2220239B1

Ferrándiz C, Liljegren SJ, Yanofsky MF (2000) Negative regulation of the SHATTERPROOF genes by FRUITFULL during Arabidopsis fruit development. Science 289:436-438

Fisher RA, Byerlee D, Edmeades G (2014) Crop yields and global food security. Australian Centre of International Agricultural Research, Canberra

Girin T, Stephenson P, Goldsack CMP, Perez A, Pires N, Sparrow PA, Wood TA, Østergaard L (2010) Brassicaceae INDEHISCENT genes specify valve margin cell fate and repress replum formation. Plant J 63:329-338 
Girin T, Paicu T, Stephenson P, Fuentes S, Körner E, O'Brien M, Sorefan K, Wood TA, Balanzá V, Ferrándiz C, Smyth DR, Østergaard L (2011) INDEHISCENT and SPATULA interact to specify carple and valve margin tissue and thus promote seed dispersal in Arabidopsis. Plant Cell 23:3641-3653

Gu Q, Ferrandiz C, Yanofsky MF, Martienssen R (1998) The FRUITFULL MADS-box gene mediates cell differentiation during Arabidopsis fruit development. Development 125:1509-1517

Hunter MC, Smith RG, Schipanski ME, Atwood LW, Mortensen DA (2017) Agriculture in 2050: recalibrating targets for sustainable intensification. Bioscience 67:386-391

Lawrenson T, Shorinola O, Stacey N, Li C, Østergaard L, Patron N, Uauy C, Harwood W (2015) Induction of targeted, heritable mutations in barley and Brassica oleracea using RNA-guided Cas9 nuclease. Genome Biol 16:258

Li X, Deb J, Kumar V, Østergaard L (2018) Temperature modulates tissue-specification program to control fruit dehiscence in Brassicaceae. Mol Plant 11:598-606

Liljegren SJ, Roeder AHK, Kempin SA, Gremski K, Østergaard L, Guimil S, Reyes DK, Yanofsky MF (2004) Control of fruit patterning in Arabidopsis by INDEHISCENT. Cell 116:843-853

Morgan CL, Bruce DM, Child R, Ladbrooke ZL, Arthur AE (1998) Genetic variation for pods resistant to shattering among lines of oilseed rape developed from synthetic $B$. napus. Field Crops Res $58: 153-165$

Morgan CL, Ladbrooke ZL, Bruce DM, Child R, Arthur AE (2000) Breeding oilseed rape for pod shattering resistance. J Agric Sci 135:347-359

Nannas NJ, Dawe RK (2015) Genetic and genomic toolbox of Zea mays. Genetics 199:655-669

Ogawa M, Kay P, Wilson S, Swain SM (2009) ARABIDOPSIS DEHISCENCE ZONE POLYGALACTURONASE1 (ADPG1), ADPG2, AND QUARTET2 are Polygalacturonases required for cell separation during reproductive development in Arabidopsis. Plant Cell 21:216-233

Østergaard L, Kempin SA, Bies D, Klee HJ, Yanofsky MF (2006) Pod shatter-resistant Brassica fruit produced by ectopic expression of the FRUITFULL gene. Plant Biotechnol J 4:45-51

Parkin IAP, Gulden SM, Sharpe AG, Lukens L, Trick M, Osborn TC, Lydiate DJ (2005) Segmental structure of the Brassica napus genome based on comparative analysis with Arabidopsis thaliana. Genetics 171:765-781

Petersen M, Sander L, van Onckelen H, Ulvskov P, Borkhardt B (1996) Isolation and characterisation of a pod dehiscence zonespecific polygalacturonase from Brassica napus. Plant Mol Biol 31:517-527

Price JS, Hobson RN, Neale MA, Bruce DM (1996) Seed losses in commercial harvesting of oilseed rape. J Agric Eng Res $80: 343-350$
Qiu D, Morgan C, Shi J, Long Y, Liu J, Li R, Zhuang X, Wang Y, Tan X, Dietrich E, Weihmann T, Everett C, Vanstraelen S, Beckett P, Fraser F, Trick M, Barnes S, Wilmer J, Schmidt R, Li J, Li D, Meng J, Bancroft I (2006) A comparative linkage map of oilseed rape and its use for QTL analysis of seed oil and erucic acid content. Theor Appl Genet 114:67-80

Rajani S, Sundaresan V (2001) The Arabidopsis myc/bHLH geneALCATRAZ enables cell separation in fruit dehiscence. Curr Biol 11:1914-1922

Raman H, Raman R, Kilian A, Detering F, Carling J et al (2014) Genome-wide delineation of natural variation for pod shatter resistance in Brassica napus. PLoS ONE 9(7):e101673

Roberts JA, Elliott KA, Gonzales-Carranza ZH (2002) Abscission, dehiscence and other cell separation processes. Ann Rev Plant Biol 53:131-158

Roeder AH, Ferrándiz C, Yanofsky MF (2003) The role of the REPLUMLESS homeodomain protein in patterning the Arabidopsis fruit. Curr Biol 13:1630-1635

Smyth DR, Bowman JL, Meyerowitz EM (1990) Early flower development in Arabidopsis. Plant Cell 2:755-767

Sorefan K, Girin T, Liljegren SJ, Ljung K, Robles P, Galván-Ampudia CS, Offringa R, Friml J, Yanofsky MF, Østergaard L (2009) A regulated auxin minimum is required for seed dispersal in Arabidopsis. Nature 459:583-586

Spence J, Vercher Y, Gates P, Harris N (1996) "Pod shatter" in Arabidopsis thaliana, Brassica napus and B. juncea. J Microsc 181:195-203

Stephenson P, Baker D, Girin T, Perez A, Amoah S, King GJ, Østergaard L (2010) A rich TILLING resource for studying gene function in Brassica rapa. BMC Plant Biol 10:62

Talon M, Koornneef M, Zeevaart JA (1990) Endogenous gibberellins in Arabidopsis thaliana and possible steps blocked in the biosynthetic pathways of the semidwarf ga4 and ga5 mutants. Proc Natl Acad Sci USA 87:7983-7987

Tilman D, Balzer C, Hill J, Befort BL (2011) Global food demand and the sustainable intensification of agriculture. Proc Natl Acad Sci USA 108:20260-20264

Uauy C (2017) Wheat genomics comes of age. Curr Opin Plant Biol 36:142-148

Wing RA, Purugganan MD, Zhang Q (2018) The rice genome revolution: from an ancient grain to Green Super Rice. Nat Rev Genet 19:505-517

Publisher's Note Springer Nature remains neutral with regard to jurisdictional claims in published maps and institutional affiliations. 